\title{
The NKG2D ligand ULBP4 is not expressed by human monocytes
}

Mariya Lazarova, Younghoon Kim, and Alexander Steinle

Institute for Molecular Medicine, Goethe-University Frankfurt am Main, 60590 Frankfurt am Main, Germany.

"correspondence to: alexander.steinle@kgu.de

ORCID Alexander Steinle: 0000-0001-5081-8503

\section{Keywords}

Innate immunity, innate lymphocytes, NK cell receptors, NKG2D, ULBP4 


\section{ABSTRACT}

The C-type lectin-like receptor NKG2D contributes to the immunosurveillance of virally infected and malignant cells by cytotoxic lymphocytes. A peculiar and puzzling feature of the NKG2D-based immunorecognition system is the high number of ligands for this single immunoreceptor. In humans, there are a total of eight NKG2D ligands (NKG2DL) comprising two members of the MIC (MICA, MICB) and six members of the ULBP family of glycoproteins (ULBP1 to ULBP6). While MICA has been extensively studied with regard to its biochemistry, cellular expression and function, very little is known about the NKG2DL ULBP4. This is, at least in part, due to its rather restricted expression by very few cell lines and tissues. Recently, constitutive ULBP4 expression by human monocytes was reported, questioning the view of tissue-restricted ULBP4 expression. Here, we scrutinized ULBP4 expression by human peripheral blood mononuclear cells and monocytes by analyzing ULBP4 transcripts and ULBP4 surface expression. In contrast to MICA, there was no ULBP4 expression detectable, neither by freshly isolated monocytes nor by PAMP-activated monocytes. However, a commercial antibody erroneously indicated surface ULBP4 on monocytes due to a non-ULBP4-specific binding activity, emphasizing the critical importance of validated reagents for life sciences. Collectively, our data show that ULBP4 is not expressed by monocytes, and likely also not by other peripheral blood immune cells, and therefore exhibits an expression pattern rather distinct from other human NKG2DL. 


\section{INTRODUCTION}

The human UL16-binding proteins (ULBPS) are a family of six MHC class I-related glycoproteins (ULBP1-6) encoded in the RAET1 gene cluster of the long arm of human chromosome 6 (Ullrich et al., 2013; Carapito and Bahram, 2015). They consist of an MHC class I-like $\alpha 1 \alpha 2$ domain anchored in the cell membrane either by a GPI-anchor (ULBP1-3, ULBP6) or via a transmembrane domain (ULBP4, ULBP5) (Cosman et al., 2001; Steinle et al., 2001; Ullrich et al., 2013; Carapito and Bahram, 2015). ULBPs together with the MHCencoded MHC class I chain-related glycoproteins A and B (MICA and MICB) constitute the ligands of the activating immunoreceptor NKG2D (Bauer et al., 1999; Cosman et al., 2001; Steinle et al., 2001; Ullrich et al., 2013; Carapito and Bahram, 2015).

NKG2D is a homodimeric C-type lectin-like receptor expressed on most human cytotoxic lymphocytes including NK cells, CD8 $\alpha \beta$ T cells and $ү \delta ~ T$ cells (Bauer et al., 1999; RinconOrozco et al., 2005). NKG2D ligation not only promotes cytolysis of NKG2D ligand (NKG2DL)-expressing target cells but also cytokine secretion by the respective NKG2D+ lymphocytes (Bauer et al., 1999; Groh et al., 2001; Jamieson et al., 2002; Rincon-Orozco et al., 2005). While NKG2DL are usually not expressed on healthy and quiescent cells, NKG2DL cell surfacing is facilitated by cellular stress, viral infection or malignant transformation, thus marking harmful cells for cytolysis (Groh et al., 1998; Raulet et al., 2013; Ullrich et al., 2013; Lanier, 2015). Various viral immune evasion mechanisms target NKG2Dmediated recognition of virus-infected cells by suppressing NKG2DL expression and surfacing (Welte et al., 2003; Lodoen and Lanier, 2005; Jonjic et al., 2008; Sester et al., 2010). NKG2D also promotes cancer immunosurveillance and allows for detection of malignant cells subjected to genotoxic stress (Raulet and Guerra, 2009; Raulet et al., 2013). However, tumors obviously evolve mechanisms to escape from NKG2D-mediated immunosurveillance, including the downregulation of NKG2DL surface expression (Eisele et al., 2006; Heinemann et al., 2012; Codo et al., 2014; Baragano Raneros et al., 2015), proteolytic shedding of NKG2DL (Salih et al., 2002; Waldhauer and Steinle, 2006; Waldhauer 
et al., 2008; Liu et al., 2010), and release of soluble NKG2DL in exosomes (Ashiru et al., 2010; Fernandez-Messina et al., 2010). Hence, arming and exploiting NKG2D-mediated cancer surveillance appears promising for therapeutic targeting of cancer. First NKG2Dbased cancer therapies are already in clinical trials (Murad et al., 2018; Baumeister et al., 2019; Lazarova and Steinle, 2019; Van Cutsem et al., 2019). However, a further in depthcharacterization of the cellular and molecular basis of the NKG2D-NKG2DL interaction and of relevant tumor escape mechanisms is critical for the rational development of novel NKG2D-based immunotherapeutic approaches.

ULBP4 is a type I transmembrane glycoprotein of the ULBP family (Chalupny et al., 2003) and one of the least characterized NKG2DL. The lack of knowledge on ULBP4 is in part also due to the scarcity of reliable detection reagents, since there are only a few commercially available "ULBP4-specific" antibodies which are in part not ULBP4-specific (Zoller et al., 2018). ULBP4 is encoded by the RAET1E gene comprising five exons, which can give rise to several alternatively spliced transcripts and ULBP4 isoforms (Carapito and Bahram, 2015; Zoller et al., 2018). Unlike ULBP1 and ULBP2, ULBP4 is not bound by the name-giving HCMV glycoprotein UL16 (Chalupny et al., 2003; Muller et al., 2010), and therefore ULBP4 can be considered as a misnomer. The few studies on ULBP4 suggest that ULBP4 expression is highly restricted and distinct from other human NKG2DL (Chalupny et al., 2003; Zoller et al., 2018). ULBP4 transcripts were detected only in a few cell lines such as HeLa cells and a few tissues such as skin and esophagus, while detection of ULBP4 glycoproteins in these cells and tissues remains a challenge up to now (Chalupny et al., 2003; Zoller et al., 2018). Recently, a constitutive ULBP4 expression on human monocytes was reported (Sharma and Markiewicz, 2019) challenging the current view of highly restricted ULBP4 expression. 


\section{RESULTS}

Recently, constitutive expression of ULBP4 on the cell surface of human monocytes was reported (Sharma and Markiewicz, 2019). In order to assess these findings, we isolated peripheral blood mononuclear cells (PBMC) from the blood of four unrelated healthy donors and analyzed ULBP4 cell surface expression of the major leukocyte subpopulations by flow cytometry, including $\mathrm{T}$ cells $\left(\mathrm{CD}^{+}\right)$, B cells $\left(\mathrm{CD} 19^{+}\right)$, NK cells $\left(\mathrm{CD} 3^{-} \mathrm{CD} 19^{-} \mathrm{NKp} 46^{+}\right)$and monocytes (CD3-CD19-CD14 ${ }^{+}$) (Figure 1A, B). ULBP4 expression was assessed with the commercially available, allegedly "ULBP4-specific" monoclonal antibody (mAb) 709116 (R\&D systems, clone 709116) utilized by Sharma et al., and with the ULBP4-specific mAb DUMO1, previously generated in our laboratory (Zoller et al., 2018). We detected a marginal binding of mAb 709116 (as compared to the isotype control) to B cells, but not to any of the other subsets of all tested donors (Figure 1B). Similarly, no DUMO1 binding was detected to any of the immune cell subsets of any of the donors. These results suggested that there are no ULBP4 molecules on the cell surface of T cells, NK cells, and monocytes. To test whether ULBP4 surface expression by monocytes may be inducible upon activation, as reported for other human NKG2DL (Kloss et al., 2008), we enriched monocytes from freshly isolated PBMC and cultured these in vitro for $16 \mathrm{~h}$ prior to flow cytometric analysis. Again, staining with DUMO1 did not detect any ULBP4 expression on cultured monocytes (Figure 1C, D). In contrast, mAb 709116 markedly bound to cultured monocytes of all four donors tested, in accord with the results reported by Sharma et al (Figure 1C, D). We also stimulated enriched monocytes with the TLR4 ligand lipopolysaccharide (LPS; at $500 \mathrm{ng} / \mathrm{mL}$ ) or with the TLR3 ligand polyinosinic:polycytidylic acid (poly(l:C); at $10 \mu \mathrm{g} / \mathrm{mL}$ ) for $16 \mathrm{~h}$ before analysis of ULBP4 surface expression. However, no DUMO1 binding was observed to any of the stimulated monocytes (data not shown).

Because of the discrepancy between the stainings of DUMO1 and mAb 709116, we addressed ULBP4 expression at the transcript level for both PBMC and enriched monocytes. To this aim, we performed qRT-PCR using primers with binding sites in exon 3 and exon 4 , 
respectively, which allow detection of all functional cell-bound ULBP4 isoforms (Figure 2A). We performed qRT-PCR for ULBP4 with cDNA from freshly isolated PBMC and from monocytes enriched from PBMC, either direct after enrichment, or after $16 \mathrm{~h}$ of in vitro cultivation either in the absence (unstimulated) or in the presence of LPS or poly(l:C), respectively. The human cervix carcinoma cell line HeLa, previously shown to express ULBP4 (Zoller et al., 2018), was used as a positive control. While ULBP4 transcripts were present in HeLa cells at substantial levels, no ULBP4 transcripts were detected neither in whole PBMC nor in any of the freshly enriched or cultured or TLR-stimulated monocytes (Figure 2B). For quality control of the experimental process, we additionally determined the expression of the NKG2DL MICA by qRT-PCR (Figure 2C). MICA transcripts were clearly detectable in all samples as expected from previous reports (Kloss et al., 2008). For an assessment of these data, it is important to add that ULBP4 transcript levels of HeLa cells do not suffice for detectable surface ULBP4 expression (Zoller et al., 2018) and that DUMO1 and mAb 709116 roughly bind with the same "strength" to ULBP4-transfected cells (Zoller et al., 2018).

Finally, we mined publicly available databases for ULBP4 transcripts in human blood cell populations (Schmiedel et al., 2018; Monaco et al., 2019; Uhlen et al., 2019). Schmiedel and colleagues analysed the RNA contents of 13 subsets of human immune cells derived from leukapheresis samples of 91 healthy donors by RNASeq. These data are publicly available in the database of immune cell expression (DICE): https://dice-database.org. In this dataset, no ULBP4 transcripts were detected in any blood cell subset except in memory CD4 T cells where extremely low levels (0.1 transcripts per million, (TPM)) were found (Schmiedel et al., 2018)(see https://dice-database.org/genes/raet1e). Monaco and colleagues provide data for 29 different types of immune cells, including various subsets of CD4 and CD8 T cells, unconventional T cells, B cells, NK cells, monocytes, dendritic cells, granulocytes and immune cell progenitors. In this dataset, extremely low levels of ULBP4 transcripts (ranging from 0.2 to 1.1 pTPM) are reported for the analyzed immune cell subsets. Data of ULBP4 transcripts, and for control of MICA transcripts, of the main immune cell populations from this 
dataset are represented in Figure 3. A third study within the framework of the human protein atlas project recently reported the transcriptome analysis for 18 immune cell subsets derived from six healthy donors (Uhlen et al., 2019). Here, ULBP4 transcripts were detected at extremely low levels in basophils, neutrophils, monocytes, T cell subsets, B cells and NK cells $(0.1$ to 0.4 pTPM). All datasets concordantly show that ULBP4 transcripts are either absent or present at extremely low levels in blood cells in general, and in monocytes, in particular. Considering that cells have been estimated to contain between 50.000 and 300.000 mRNA molecules (Marinov et al., 2014), an abundance of 0.1 to 1 TPM as determined for ULBP4 in various immune subsets would accordingly correspond to one ULBP4 transcript per $3-180$ cells. Obviously, such expression would neither result in detectable surface expression nor translate into biological significance for a given immune cell subset. 


\section{CONCLUDING REMARKS}

The validity of experimental data strongly depends on the quality and specificity of the reagents used. Here we report on another unfortunate example how a cross-reactive antibody does cause misinterpretation of experimental data. The commercially available "ULBP4 specific" mAb 709116 binds to human monocytes (our data and (Sharma and Markiewicz, 2019)). However, both the qRT-PCR data presented here and datasets of three independent databases show that there are virtually no ULBP4 transcripts in human monocytes. Lack of ULBP4 surface expression by monocytes was also confirmed by the validated ULBP4-specific mAb DUMO1. Obviously, mAb 709116 cross-reacts with a surface molecule on human cells, which is not ULBP4, as already stated previously (Zoller et al., 2018). This emphasizes that experimental data obtained with the cross-reactive mAb 709116 antibody must be interpreted with caution and require validation by independent methods. Collectively, the presented data exclude that ULBP4 is expressed by human monocytes under normal conditions and upon activation by PAMP. In addition, our data together with public available data sets also strongly suggest that ULBP4 is not expressed by peripheral blood mononuclear cells in contrast to other human NKG2DL. These data support the notion of ULBP4 fulfilling a non-redundant, tissue-specific function within the human immune system that awaits elucidation by future studies. 


\section{MATERIALS AND METHODS}

\section{PBMC isolation and monocyte enrichment}

Blood samples were obtained from four healthy donors with approval of the local Ethics Committee and PBMC were isolated via density gradient centrifugation with Ficoll Paque Plus (GE Healthcare, Chicago, IL, USA). Monocytes were enriched by negative selection using a pan monocyte isolation kit (MiltenyiBiotec, Bergisch Gladbach, Germany) according to the manufacturer's protocol.

\section{Cell culture}

Monocytes were cultured in RPMI 1640 medium (Sigma-Aldrich, St. Louis, MO, USA) supplemented with 10\% fetal calf serum (FCS) (Biochrome, Berlin, Germany), 1 mM sodium pyruvate (Thermo Fisher, Waltham, MA, USA), $2 \mathrm{mM} \mathrm{L-glutamine} \mathrm{and} 100 \mathrm{U} / \mathrm{ml}$ penicillin/100 mg/ml streptomycin (Sigma-Aldrich). For activation, media were supplemented with $500 \mathrm{ng} / \mathrm{mL}$ LPS (Sigma-Aldrich, St. Louis, MO, USA) or with $10 \mu \mathrm{g} / \mathrm{mL}$ poly(l:C) (Invitrogen, Carlsbad, CA, USA). HeLa cells were cultured in Dulbecco's Modified Eagle Medium (Thermo Fisher Scientific, Waltham, MA, USA) supplemented with $10 \%$ FCS (Biochrome, Berlin, Germany), $2 \mathrm{mM}$ L-glutamine and $100 \mathrm{U} / \mathrm{ml}$ penicillin $/ 100 \mathrm{mg} / \mathrm{ml}$ streptomycin (Sigma-Aldrich).

\section{Antibodies}

Anti-ULBP4 mAb (clone 709116) was purchased from R\&D (Minneapolis, MN, USA). The ULBP4-specific mAb DUMO1 was previously described (Zoller et al., 2018). Anti-CD3PerCP-Cy5.5 and fixable viability dye(FVD)-eFluor506 were from eBioscience (SanDiego, CA, USA), anti-CD19-APC-Cy7, anti-CD14-FITC, anti-NKp46-PE-Cy7, streptavidin-BV421 
(SA-BV421) and goat-anti-mouse-IgG-PE (GaM-PE) were from Biolegend (SanDiego, CA, USA).

\section{Flow cytometry}

Cells were first incubated with $30 \mu \mathrm{g} / \mathrm{mL}$ human IgG to block Fc-receptors for 20 min at $4^{\circ} \mathrm{C}$, and subsequently with the indicated $\mathrm{mAb}(10 \mu \mathrm{g} / \mathrm{ml})$ for another $20 \mathrm{~min}$ at $4^{\circ} \mathrm{C}$. After washing, cell bound antibodies were stained with fluorochrome-conjugated goat anti-mouse IgG antibodies (Jackson ImmunoResearch). PBMC were subsequently stained with anti-CD3PerCP-Cy5.5, anti-CD19-APC-Cy7, anti-CD14-FITC and anti-NKp46-PE-Cy7. Dead cells were excluded from the analysis by staining with fixable viability dye efluor ${ }^{\mathrm{TM}} 450$ (eBioscience). Flow cytometric analysis was performed on a FACS Canto II (BD Biosciences, Heidelberg, Germany) and data analyzed with FlowJo software (TreeStar, Ashland, Oh, USA).

\section{Quantitative RT-PCR (qRT-PCR)}

RNA was isolated from pelleted cells with the RNAqueous Micro Total RNA isolation kit (Invitrogen) according to the manufacturer's protocol. Subsequently, RNA was treated with RNAse-free DNAse (Promega, Madison, WI, USA) and converted into cDNA using random primers (Promega) and M-MLV RT RNAse (H-) Point Mutant (Promega) according to the manufacturer's protocol. For quantification of ULBP4 and MICA transcripts, cDNA was amplified with ULBP4-specific (forward: 5'-ctg gct cag gga att ctt agg-3'; reverse: 5'-cta gaa gaa gac cag tgg ata tc-3') or MICA-specific primer pairs (forward: 5'-cct tgg cca tga acg tca gg-3'; reverse: 5'-cct ctg agg cct cac tgc g-3'). For normalization, 18S rRNA was detected (forward: 5'-cgg cta cca cat cca agg aa-3'; reverse: 5'-gct gga att acc gcg gct-3'). For positive control and normalisation, transcript levels of HeLa cells were determined. Quantitative PCR was performed with SYBR Green (Roche, Basel, Switzerland) on a QuantStudio 3 system 
(Applied Biosystems, Foster City, CA, USA). Relative gene expression normalized to $18 \mathrm{~S}$ rRNA was calculated with the $\Delta \Delta$ CT method.

\section{ACKNOWLEDGEMENTS}

We would like to thank Christina Born, Nikita Verheyden, and Ines Kühnel for technical support and advice. 


\section{REFERENCES}

Ashiru, O., Boutet, P., Fernandez-Messina, L., Aguera-Gonzalez, S., Skepper, J.N., ValesGomez, M., et al. (2010). Natural killer cell cytotoxicity is suppressed by exposure to the human NKG2D ligand MICA*008 that is shed by tumor cells in exosomes. Cancer Res 70(2), 481-489. doi: 10.1158/0008-5472.CAN-09-1688.

Baragano Raneros, A., Martin-Palanco, V., Fernandez, A.F., Rodriguez, R.M., Fraga, M.F., Lopez-Larrea, C., et al. (2015). Methylation of NKG2D ligands contributes to immune system evasion in acute myeloid leukemia. Genes Immun 16(1), 71-82. doi: 10.1038/gene.2014.58.

Bauer, S., Groh, V., Wu, J., Steinle, A., Phillips, J.H., Lanier, L.L., et al. (1999). Activation of NK cells and T cells by NKG2D, a receptor for stress-inducible MICA. Science 285(5428), 727-729. doi: 10.1126/science.285.5428.727.

Baumeister, S.H., Murad, J., Werner, L., Daley, H., Trebeden-Negre, H., Gicobi, J.K., et al. (2019). Phase I Trial of Autologous CAR T Cells Targeting NKG2D Ligands in Patients with AML/MDS and Multiple Myeloma. Cancer Immunol Res 7(1), 100-112. doi: 10.1158/2326-6066.CIR-18-0307.

Carapito, R., and Bahram, S. (2015). Genetics, genomics, and evolutionary biology of NKG2D ligands. Immunol Rev 267(1), 88-116. doi: 10.1111/imr.12328.

Chalupny, N.J., Sutherland, C.L., Lawrence, W.A., Rein-Weston, A., and Cosman, D. (2003). ULBP4 is a novel ligand for human NKG2D. Biochem Biophys Res Commun 305(1), 129-135. doi: 10.1016/s0006-291x(03)00714-9.

Codo, P., Weller, M., Meister, G., Szabo, E., Steinle, A., Wolter, M., et al. (2014). MicroRNAmediated down-regulation of NKG2D ligands contributes to glioma immune escape. Oncotarget 5(17), 7651-7662. doi: 10.18632/oncotarget.2287.

Cosman, D., Mullberg, J., Sutherland, C.L., Chin, W., Armitage, R., Fanslow, W., et al. (2001). ULBPs, novel MHC class I-related molecules, bind to CMV glycoprotein UL16 
and stimulate NK cytotoxicity through the NKG2D receptor. Immunity 14(2), 123-133. doi: 10.1016/s1074-7613(01)00095-4.

Eisele, G., Wischhusen, J., Mittelbronn, M., Meyermann, R., Waldhauer, I., Steinle, A., et al. (2006). TGF-beta and metalloproteinases differentially suppress NKG2D ligand surface expression on malignant glioma cells. Brain 129(Pt 9), 2416-2425. doi: 10.1093/brain/awl205.

Fernandez-Messina, L., Ashiru, O., Boutet, P., Aguera-Gonzalez, S., Skepper, J.N., Reyburn, H.T., et al. (2010). Differential mechanisms of shedding of the glycosylphosphatidylinositol (GPI)-anchored NKG2D ligands. J Biol Chem 285(12), 8543-8551. doi: 10.1074/jbc.M109.045906.

Groh, V., Rhinehart, R., Randolph-Habecker, J., Topp, M.S., Riddell, S.R., and Spies, T. (2001). Costimulation of CD8alphabeta T cells by NKG2D via engagement by MIC induced on virus-infected cells. Nat Immunol 2(3), 255-260. doi: 10.1038/85321.

Groh, V., Steinle, A., Bauer, S., and Spies, T. (1998). Recognition of stress-induced MHC molecules by intestinal epithelial gammadelta T cells. Science 279(5357), 1737-1740. doi: 10.1126/science.279.5357.1737.

Heinemann, A., Zhao, F., Pechlivanis, S., Eberle, J., Steinle, A., Diederichs, S., et al. (2012). Tumor suppressive microRNAs miR-34a/c control cancer cell expression of ULBP2, a stress-induced ligand of the natural killer cell receptor NKG2D. Cancer Res 72(2), 460-471. doi: 10.1158/0008-5472.CAN-11-1977.

Jamieson, A.M., Diefenbach, A., McMahon, C.W., Xiong, N., Carlyle, J.R., and Raulet, D.H. (2002). The role of the NKG2D immunoreceptor in immune cell activation and natural killing. Immunity 17(1), 19-29. doi: 10.1016/s1074-7613(02)00333-3.

Jonjic, S., Polic, B., and Krmpotic, A. (2008). Viral inhibitors of NKG2D ligands: friends or foes of immune surveillance? Eur J Immunol 38(11), 2952-2956. doi: 10.1002/eji.200838823. 
Kloss, M., Decker, P., Baltz, K.M., Baessler, T., Jung, G., Rammensee, H.G., et al. (2008). Interaction of monocytes with NK cells upon Toll-like receptor-induced expression of the NKG2D ligand MICA. J Immunol 181(10), 6711-6719. doi: 10.4049/jimmunol.181.10.6711.

Lanier, L.L. (2015). NKG2D Receptor and Its Ligands in Host Defense. Cancer Immunol Res 3(6), 575-582. doi: 10.1158/2326-6066.CIR-15-0098.

Lazarova, M., and Steinle, A. (2019). The NKG2D axis: an emerging target in cancer immunotherapy. Expert Opin Ther Targets 23(4), 281-294. doi: 10.1080/14728222.2019.1580693.

Liu, G., Atteridge, C.L., Wang, X., Lundgren, A.D., and Wu, J.D. (2010). The membrane type matrix metalloproteinase MMP14 mediates constitutive shedding of MHC class I chain-related molecule $A$ independent of $A$ disintegrin and metalloproteinases. $J$ Immunol 184(7), 3346-3350. doi: 10.4049/jimmunol.0903789.

Lodoen, M.B., and Lanier, L.L. (2005). Viral modulation of NK cell immunity. Nat Rev Microbiol 3(1), 59-69. doi: 10.1038/nrmicro1066.

Marinov, G.K., Williams, B.A., McCue, K., Schroth, G.P., Gertz, J., Myers, R.M., et al. (2014). From single-cell to cell-pool transcriptomes: stochasticity in gene expression and RNA splicing. Genome Res 24(3), 496-510. doi: 10.1101/gr.161034.113.

Monaco, G., Lee, B., Xu, W., Mustafah, S., Hwang, Y.Y., Carre, C., et al. (2019). RNA-Seq Signatures Normalized by mRNA Abundance Allow Absolute Deconvolution of Human Immune Cell Types. Cell Rep 26(6), $1627-1640$ e1627. doi: 10.1016/j.celrep.2019.01.041.

Muller, S., Zocher, G., Steinle, A., and Stehle, T. (2010). Structure of the HCMV UL16-MICB complex elucidates select binding of a viral immunoevasin to diverse NKG2D ligands. PLoS Pathog 6(1), e1000723. doi: 10.1371/journal.ppat.1000723. 
Murad, J.M., Baumeister, S.H., Werner, L., Daley, H., Trebeden-Negre, H., Reder, J., et al. (2018). Manufacturing development and clinical production of NKG2D chimeric antigen receptor-expressing $\mathrm{T}$ cells for autologous adoptive cell therapy. Cytotherapy 20(7), 952-963. doi: 10.1016/j.jcyt.2018.05.001.

Raulet, D.H., Gasser, S., Gowen, B.G., Deng, W., and Jung, H. (2013). Regulation of ligands for the NKG2D activating receptor. Annu Rev Immunol 31, 413-441. doi: 10.1146/annurev-immunol-032712-095951.

Raulet, D.H., and Guerra, N. (2009). Oncogenic stress sensed by the immune system: role of natural killer cell receptors. Nat Rev Immunol 9(8), 568-580. doi: 10.1038/nri2604.

Rincon-Orozco, B., Kunzmann, V., Wrobel, P., Kabelitz, D., Steinle, A., and Herrmann, T. (2005). Activation of $\mathrm{V}$ gamma 9V delta $2 \mathrm{~T}$ cells by NKG2D. J Immunol 175(4), 21442151. doi: 10.4049/jimmunol.175.4.2144.

Salih, H.R., Rammensee, H.G., and Steinle, A. (2002). Cutting edge: down-regulation of MICA on human tumors by proteolytic shedding. J Immunol 169(8), 4098-4102. doi: 10.4049/jimmunol.169.8.4098.

Schmiedel, B.J., Singh, D., Madrigal, A., Valdovino-Gonzalez, A.G., White, B.M., ZapardielGonzalo, J., et al. (2018). Impact of Genetic Polymorphisms on Human Immune Cell Gene Expression. Cell 175(6), 1701-1715 e1716. doi: 10.1016/j.cell.2018.10.022.

Sester, M., Koebernick, K., Owen, D., Ao, M., Bromberg, Y., May, E., et al. (2010). Conserved amino acids within the adenovirus $2 \mathrm{E} 3 / 19 \mathrm{~K}$ protein differentially affect downregulation of MHC class I and MICA/B proteins. J Immunol 184(1), 255-267. doi: 10.4049/jimmunol.0902343.

Sharma, N., and Markiewicz, M.A. (2019). Constitutive expression of ULBP-4 on monocytes regulates NK cell NKG2D expression. Blood Adv 3(10), 1563-1567. doi: 10.1182/bloodadvances.2018025742. 
Steinle, A., Li, P., Morris, D.L., Groh, V., Lanier, L.L., Strong, R.K., et al. (2001). Interactions of human NKG2D with its ligands MICA, MICB, and homologs of the mouse RAE-1 protein family. Immunogenetics 53(4), 279-287. doi: 10.1007/s002510100325.

Uhlen, M., Karlsson, M.J., Zhong, W., Tebani, A., Pou, C., Mikes, J., et al. (2019). A genomewide transcriptomic analysis of protein-coding genes in human blood cells. Science 366(6472). doi: 10.1126/science.aax9198.

Ullrich, E., Koch, J., Cerwenka, A., and Steinle, A. (2013). New prospects on the NKG2D/NKG2DL system for oncology. Oncoimmunology 2(10), e26097. doi: 10.4161/onci.26097.

Van Cutsem, E., Machiels, J., Van den Eynde, M., Prenen, H., Hendlisz, A., Shaza, L., et al. (2019). Phase 1 studies assessing the safety and clinical activity of autologous and allogeneic NKG2D-based CAR-T therapy in metastatic colorectal cancer. Ann Oncol 30 Suppl 4, iv124-iv125. doi: 10.1093/annonc/mdz157.008.

Waldhauer, I., Goehlsdorf, D., Gieseke, F., Weinschenk, T., Wittenbrink, M., Ludwig, A., et al. (2008). Tumor-associated MICA is shed by ADAM proteases. Cancer Res 68(15), 6368-6376. doi: 10.1158/0008-5472.CAN-07-6768.

Waldhauer, I., and Steinle, A. (2006). Proteolytic release of soluble UL16-binding protein 2 from tumor cells. Cancer Res 66(5), 2520-2526. doi: 10.1158/0008-5472.CAN-052520.

Welte, S.A., Sinzger, C., Lutz, S.Z., Singh-Jasuja, H., Sampaio, K.L., Eknigk, U., et al. (2003). Selective intracellular retention of virally induced NKG2D ligands by the human cytomegalovirus UL16 glycoprotein. Eur J Immunol 33(1), 194-203. doi: 10.1002/immu.200390022.

Zoller, T., Wittenbrink, M., Hoffmeister, M., and Steinle, A. (2018). Cutting an NKG2D Ligand Short: Cellular Processing of the Peculiar Human NKG2D Ligand ULBP4. Front Immunol 9, 620. doi: 10.3389/fimmu.2018.00620. 


\section{FIGURE LEGENDS}

\section{Figure 1: Flow cytometric analysis of ULBP4 cell surface expression by human} monocytes and other PBMC subsets. (A, B) Freshly isolated human PBMC were analyzed by flow cytometry for ULBP4 surface expression using mAb DUMO1 or mAb 709116 with

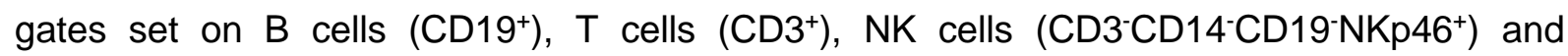
monocytes (CD3-CD14 $\left.{ }^{+} \mathrm{CD} 19^{-} \mathrm{NKp} 46^{-}\right)$, respectively. (A) Representative gating strategy. (B) Representative histogram overlays of ULBP4 stainings (solid line) and isotype controls (gray filled). (C) Monocytes enriched from PBMC were cultured for $16 \mathrm{~h}$ and subsequently analyzed by flow cytometry for ULBP4 surface expression with DUMO1 or 709116. Representative gating of cultured $\mathrm{CD} 14^{+}$cells (left) and overlays of DUMO1 or 709116 stainings (solid lines), respectively, with isotype controls (gray). (D) Comprehensive depiction of ULBP4 surface expression on either monocytes (CD3-CD14+CD19-NKp46-) among freshly isolated PBMC (left) or after $16 \mathrm{~h}$ culture (right) of four human healthy donors. Specific fluorescence intensity (SFI) is shown (SFI = MFI (ULBP4 mAb) - MFI (isotype control)). n. d. $=$ not detectable.

Figure 2: No ULBP4 transcripts detectable in human PBMC and monocytes. (A) Schematic localization of PCR primer sites (Ex3fw, Ex4rv) of the ULBP4-specific qRT-PCR within in the ULBP4-encoding RAET1E gene locus. The primer pair was designed to detect all known ULBP4 isoforms transcripts. (B) There were no ULBP4 transcripts detectable by qRT-PCR in all PBMC-derived samples of four human donors including freshly isolated PBMC, enriched monocytes, enriched monocytes after $16 \mathrm{~h}$ culture without stimulation (-) or with lipopolysaccharide (LPS) (at $500 \mathrm{ng} / \mathrm{mL}$ ) or with polyinosinic:polycytidylic acid (poly(l:C)( at $10 \mu \mathrm{g} / \mathrm{mL}$ ). HeLa cells were used as positive control. (C) All samples were also analyzed by qRT-PCR for MICA transcripts for comparison and to control for quality of cDNA preparation. $(B, C)$ Expression levels were normalized to $18 \mathrm{~S}$ rRNA and set relative to the transcript levels of HeLa cells. 


\section{Figure 3: Mining of RNASeq datasets for human PBMC subsets reveal no or minimal}

ULBP4 expression. (A) Number of ULBP4 transcripts (pTPM: transcripts per million) and (B), for comparison, of MICA transcripts in several immune cell subsets, including various monocyte subsets. Data were reported by Monaco et al (Monaco et al., 2019) and retrieved via the human protein atlas (https://www.proteinatlas.org). 


$$
\frac{1}{1} \frac{1}{1}
$$




\section{ULBP4}
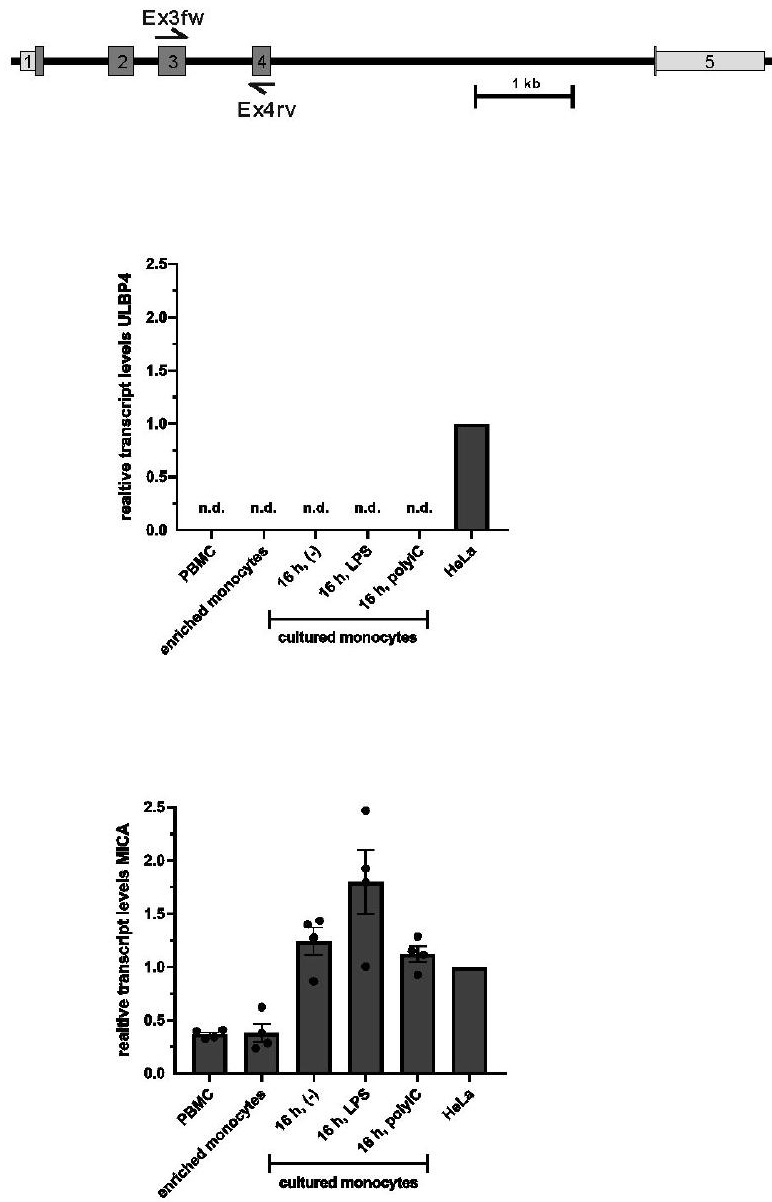

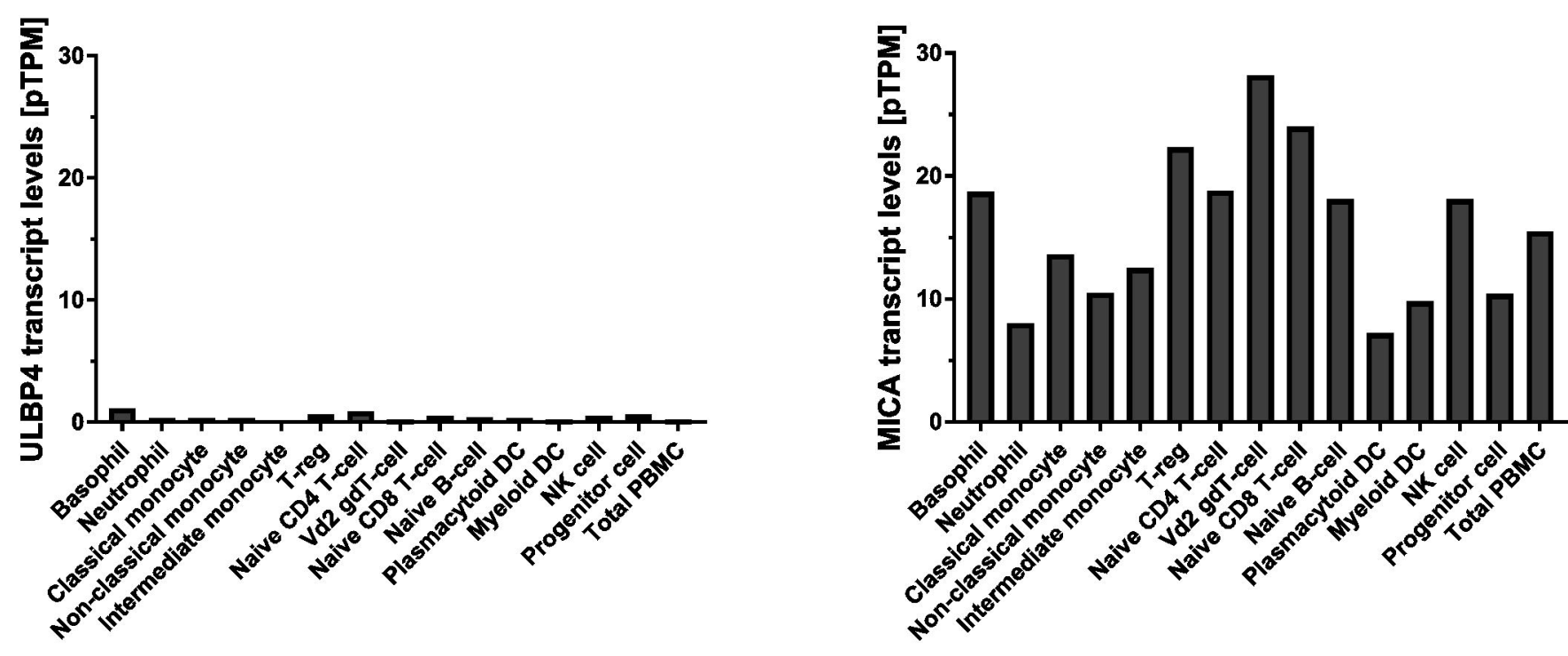

Figure 3 